\title{
OPEN Co-feeding glucose with either gluconate or galacturonate during clostridial fermentations provides metabolic fine-tuning capabilities
}

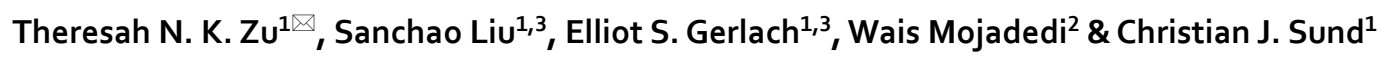

Clostridium acetobutylicum ATCC 824 effectively utilizes a wide range of substrates to produce commodity chemicals. When grown on substrates of different oxidation states, the organism exhibits different recycling needs of reduced intracellular electron carrying co-factors. Ratios of substrates with different oxidation states were used to modulate the need to balance electron carriers and demonstrate fine-tuned control of metabolic output. Three different oxidized substrates were first fed singularly, then in different ratios to three different strains of Clostridium sp. Growth was most robust when fed glucose in exclusive fermentations. However, the use of the other two more oxidized substrates was strain-dependent in exclusive feeds. In glucose-galacturonate mixed fermentation, the main products (acetate and butyrate) were dependant on the ratios of the substrates. Exclusive fermentation on galacturonate was nearly homoacetic. Co-utilization of galacturonate and glucose was observed from the onset of fermentation in growth conditions using both substrates combined, with the proportion of galacturonate present dictating the amount of acetate produced. For all three strains, increasing galacturonate content (\%) in a mixture of galacturonate and glucose from 0 to 50, and 100 , resulted in a corresponding increase in the amount of acetate produced. For example, $C$. acetobutylicum increased from $\sim 10 \mathrm{mM}$ to $\sim 17 \mathrm{mM}$, and then $\sim 23 \mathrm{mM}$. No co-utilization was observed when galacturonate was replaced with gluconate in the two substrate co-feed.

Biology is inherently capable of producing certain high-value products, where synthetic chemistry struggles ${ }^{1,2}$. Synthesis of chemicals using microorganisms requires complex, intensive, and time consuming metabolic engineering. However, chemical production pipelines are executable as discrete components that are quickly inserted or deleted ${ }^{3,4}$. This bioengineering step provides the necessary pathway flux for the desired chemical production. However, it is challenging to know the correct amount of a particular carbon substrate and redox co-factors of the right oxidation state for enabling enzymes of a specific desired pathway in the context of background cellular metabolism. Current available technologies or processes are not amenable to complex inputs ${ }^{5}$. Traditionally, single substrates, most notably sugars, such as glucose, have been explored as substrates, leading to several limitations on the metabolism of cells ${ }^{6}$. In contrast, providing microbes with alternate substrates has the potential to ease such constraints and mitigate issues by adding an extra degree of freedom to the cellular systems and can allow for optimization of fermentation products ${ }^{6}$.

Advances in synthetic biology are unlocking the potential to produce a vast array of chemicals with microorganisms ${ }^{7-9}$. Chemicals production via synthetic pathways imposes a metabolic burden on cells since resources, in the form of carbon and energy, are re-directed towards the desired product ${ }^{6,10,11}$. Maximizing product yields without sacrificing the microbial cells that serve as product factories is essential for sustained and economical chemical production ${ }^{12,13}$. Synthetic and natural pathways require exact proportions of precursors such as carbon, ATP, and electron carriers to produce the desired chemical ${ }^{6,14}$. Generally, product synthesis in cells occurs at varying efficiency levels that involves the coordination of different biological building blocks. In particular, exclusively fed, substrates produce specific carbon-to-energy ratios that are different from carbon-to-energy

${ }^{1}$ Combat Capabilities Development Command Army Research Laboratory, SEDD, Adelphi, MD 20783, USA. ${ }^{2}$ Oak Ridge Associated Universities, Belcamp, MD 21017, USA. ${ }^{3}$ These authors contributed equally: Sanchao Liu and Elliot S. Gerlach. ${ }^{\boxplus}$ email:Theresah.Zu.civ@mail.mil 
requirements of desired products ${ }^{15}$. Surplus precursors are metabolically wasteful and deprive the cell of precious resources that could be re-directed toward growth and maintenance ${ }^{6}$. This excess input can lead to a production of undesired products ${ }^{16,17}$. Current strategies at re-direction of these excess inputs have focused on the generation of biosynthetic pathways ${ }^{18-20}$ and the reconfiguration of bioreactors ${ }^{21}$. However, less consideration has gone into the optimization of precursor production in cells. A method to provide exact precursor proportions for synthetic pathways would eliminate metabolic waste and, in turn, increase chemical yields.

Anaerobic clostridia have a long history of acetone, butanol, and ethanol production from carbohydrate-rich substrates in ABE fermentation. Anaerobic fermentation has promise as an alternative solution for energy recycling of food waste, which generates approximately $30-60 \%$ of the total solid waste globally ${ }^{22}$. In military environments, challenges with generated food waste impose an additional logistical burden on Army operations ${ }^{23,24}$, with current guidelines including burial or active burning of any generated food waste ${ }^{25}$. These clostridia can utilize a wide range of carbohydrates found in lignocellulosic biomass, which is readily available as agricultural, agro-industrial, and food waste ${ }^{26}$. Plant biomass found in agricultural lignocellulosic biomass contains pectin, a carbohydrate polymer made up of glucose, $\mathrm{L}$-arabinose, and D-galacturonate ${ }^{27}$. D-Galacturonate and D-gluconate (present in fruit) are oxidized carbohydrate derivatives, which have been the focused substrates in previous $C$. acetobutylicum studies ${ }^{28}$. To advance food waste fermentation processes, an understanding of the metabolism of these oxidized substrates is essential. Our lab observed homoacetic fermentation on galacturonate in previous research, suggesting a potential route for biologically derived acetic acid production ${ }^{28}$. Advances in genetic engineering techniques ${ }^{29,30}$ in clostridia, during the last decade, have unlocked the potential of inserting synthetic pathways for chemical production into these organisms. The solventogenic clostridia are excellent synthetic biology chassis candidates because of their wide substrate range, high level of carbon flux through acetyl-CoA, and demonstrated ability to produce chemicals at scale ${ }^{31}$.

The high level of flux through acetyl-CoA is particularly important because it can serve as the precursor to the synthesis of many classes of compounds such as acetate, ethanol, lactate, butyrate, butanol, and acetone ${ }^{32-34}$. Cofeeding of substrates to solventogenic clostridium is a possible mechanism to control proportions of ATP, NADH, and acetyl-CoA ${ }^{35}$. A barrier to co-feeding is carbon catabolite repression (CCR), which occurs widely in nature and prevents co-utilization if preferred substrates are present ${ }^{33}$. CCR occurs in the solventogenic clostridium were glucose, and in some cases, arabinose represses the utilization of less preferred carbohydrates ${ }^{36-38}$. Many clostridia can consume substrates that differ from carbohydrates in the oxidation state. These include aldonic acids, uronic acids, sugar alcohols, and glycerol. This capability can be exploited for fine-tuned metabolic control. However, there is a lack of information about the co-utilization of these substrates and regulatory processes, which would prevent co-utilization.

In this study, we examine the ability to fine-tune the metabolism of Clostridium sp. by co-feeding the organism a mix of glucose together with either of two more oxidized substrates. By varying the proportion of galacturonate in the mixture, it was possible to fine-tune control of reduced electron carrier production, thereby enabling optimization of redox inputs to particular chemical production pathways without genetic alterations. This approach bypasses the limitations of conventional techniques or solutions that generally require pathway modification via metabolic engineering ${ }^{12}$, which tend to be laborious processes without guarantee of the desired output or result in other negative side-effects. The ability to use native pathways to exert metabolic control through the choice of controlled substrate, explained here, decreases the risks and costs associated with direct metabolic engineering.

\section{Results}

Control of metabolic state of fermentative organisms. Fermentative organisms such as Clostridium sp. adjust their metabolic state to make the most efficient use of substrates with different oxidation states. They do this by altering the recycling of reduced electron carriers such as NADH and NADPH. In general, the higher the oxidation state of a substrate, the less $\mathrm{NAD}(\mathrm{P}) \mathrm{H}$ it generates in its catabolism ${ }^{35}$. During homoacetic fermentation on galacturonate ${ }^{28}$, the batch fermentation was thought to be incomplete regardless of product inhibition or low substrate utilization. Also, galacturonate-grown cells exhibited reduced growth rates when the fermentation culture was agitated, suggesting loss in $\mathrm{H}_{2}$ reductant, required for redox balancing. A detailed pathway of these carriers when C. acetobutylicum is fed three substrates (galacturonate, gluconate, glucose) of different oxidation states is presented in Fig. 1, below. The figure is an updated pathway from an earlier publication which includes pathways for mannitol and gylcerol utilization ${ }^{38,39}$. Figure 1 shows that from uptake to acetyl$\mathrm{CoA}$ a combination of $0.3 \mathrm{ATP} / 0 \mathrm{NADH}$ is produced during galacturonate utilization, $0.3 \mathrm{ATP} / 0.5 \mathrm{NADH}$ for gluconate, $1 \mathrm{ATP} / 1 \mathrm{NADH}$ for glucose, $1 \mathrm{ATP} / 1.5 \mathrm{NADH}$ for mannitol, and $0.6 \mathrm{ATP} / 2 \mathrm{NADH}$ for glycerol. These ratios thus present discreet points of operation during individual substrate feeding.

Growth on singular and mixed substrates. One strain, C. acetobutylicum, was initially tested in experiments for utilization of substrates during the acidogenic phase of the fermentation. C. acetobutylicum cells were cultured following the 13 substrate combinations (minus combinations of 75\%/25\%) in Table 1 below. From the $\mathrm{OD}_{600}$ (Supplementary Figure S1a) and metabolites data (supplementary Figure S2a-c), individual substrate feeding suggests glucose was the most readily used of the three substrates with cells utilizing (producing) $\sim 25 \mathrm{mM}$ glucose $(\sim 14 \mathrm{mM}$ butyrate, $\sim 10 \mathrm{mM}$ acetate $), \sim 19 \mathrm{mM}$ gluconate $(\sim 17 \mathrm{mM}$ acetate and $\sim 6 \mathrm{mM}$ butyrate), and $\sim 15 \mathrm{mM}$ galacturonate ( $\sim 23 \mathrm{mM}$ acetate as the sole product) within the first $8 \mathrm{~h}$ of fermentation. In order of decreasing oxidation state, glucose was the most reduced substrate, followed by gluconate and then galacturonate, with the most oxidized substrate being nearly homoacetic. Gluconate though used more readily than galacturonate, resulted in a substantial decrease in cell growth rate indicated by the $\mathrm{OD}_{600}$ data with $C$. acetobutylicum being the most tolerant strain. On the other hand, growth on galacturonate was robust for $C$. saccharoperbutylacetonicum and C. beijerinckii and less robust for C. acetobutylicum. 


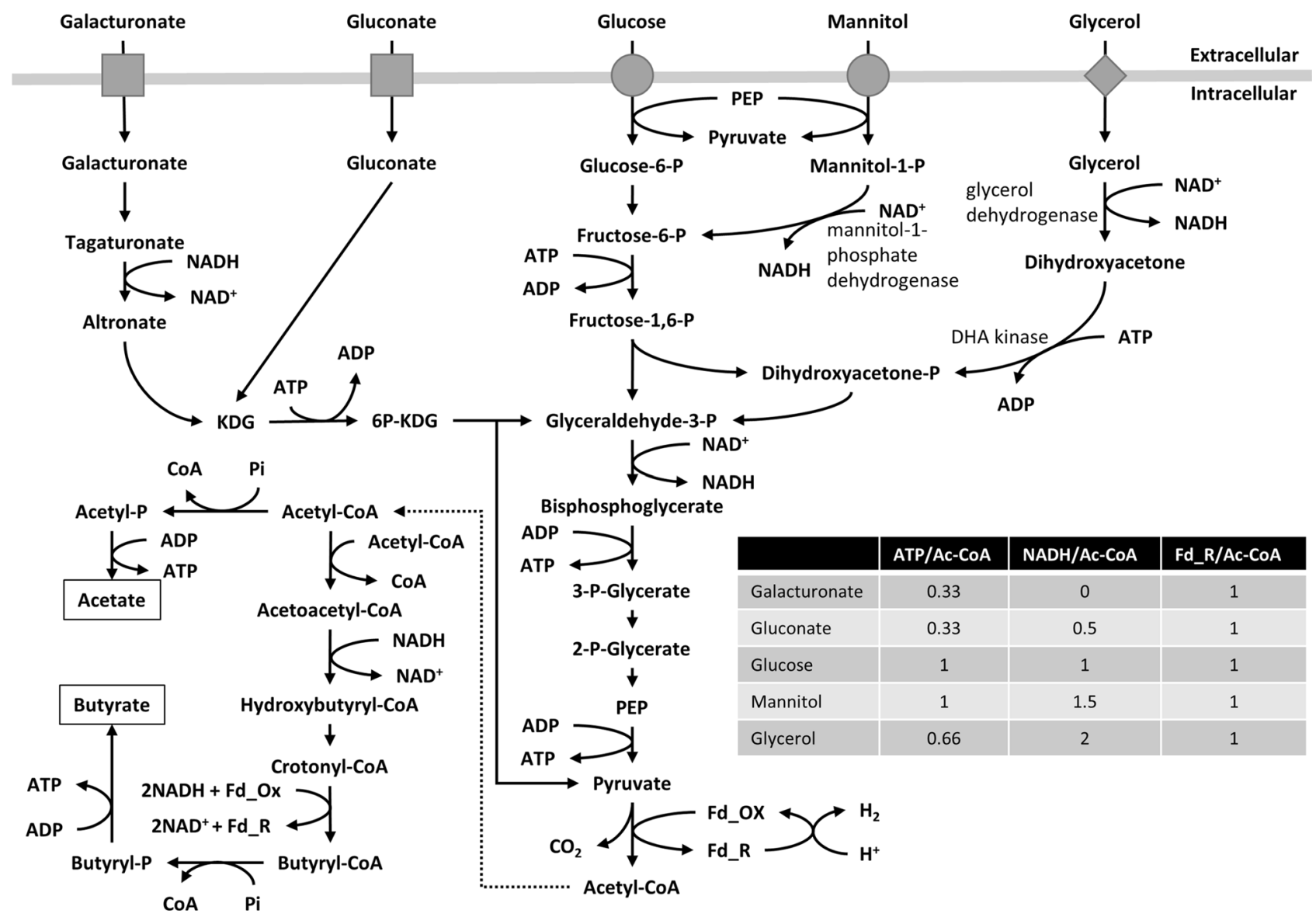

Figure 1. Metabolic network of Clostridium acetobutylicum. Updated pathway from original published work (Servinsky et al. ${ }^{28}$ ).

\begin{tabular}{|c|c|c|c|c|c|}
\hline \multicolumn{6}{|c|}{ Experimental conditions } \\
\hline \multirow[b]{2}{*}{ Available substrates } & \multicolumn{3}{|c|}{$\% \mathrm{C}$ in co-feed } & \multicolumn{2}{|c|}{$\begin{array}{l}\text { Net NADH per acetyl-CoA } \\
\text { from substrate to product }\end{array}$} \\
\hline & \begin{tabular}{|l} 
Glucose \\
\end{tabular} & Gluconate & Galacturonate & Acetate & Butyrate \\
\hline$\# 1$ & 100 & 0 & 0 & 1 & -0.50 \\
\hline$\# 2$ & 75 & 0 & 25 & 0.75 & -0.75 \\
\hline$\# 3$ & 50 & 0 & 50 & 0.50 & -1 \\
\hline$\# 4$ & 25 & 0 & 75 & 0.25 & -1.25 \\
\hline$\# 5$ & 0 & 0 & 100 & 0 & -1.50 \\
\hline$\# 6$ & 0 & 25 & 75 & 0.13 & -1.138 \\
\hline$\# 7$ & 0 & 50 & 50 & 0.25 & -1.25 \\
\hline$\# 8$ & 0 & 75 & 25 & 0.38 & -1.13 \\
\hline$\# 9$ & 0 & 100 & 0 & 0.50 & -1 \\
\hline$\# 10$ & 25 & 75 & 0 & 0.63 & -0.88 \\
\hline$\# 11$ & 50 & 50 & 0 & 0.75 & -0.75 \\
\hline$\# 12$ & 75 & 25 & 0 & 0.88 & -0.63 \\
\hline$\# 13$ & 33.33 & 33.33 & 33.33 & 0.50 & -0.99 \\
\hline
\end{tabular}

Table 1. Combinations of three substrates with different oxidation states for Clostridium sp. fermentations. Also shown are the net NADH per acetyl CoA for production of the two main fermentation products - acetate and butyrate. Values for growth on individual substrate feeding are shown in bold, whereas predicted outcomes for co-feeds are shown in italic. In general growth on these substrates resulted in product mixtures required for redox balancing. The negative values indicate that undesirable by-products are generated during this process. So in general, targeted butyrate production will always have associated acetate as a by-product for electron balancing. 
a Co-utilization in C. acetobutylicum b
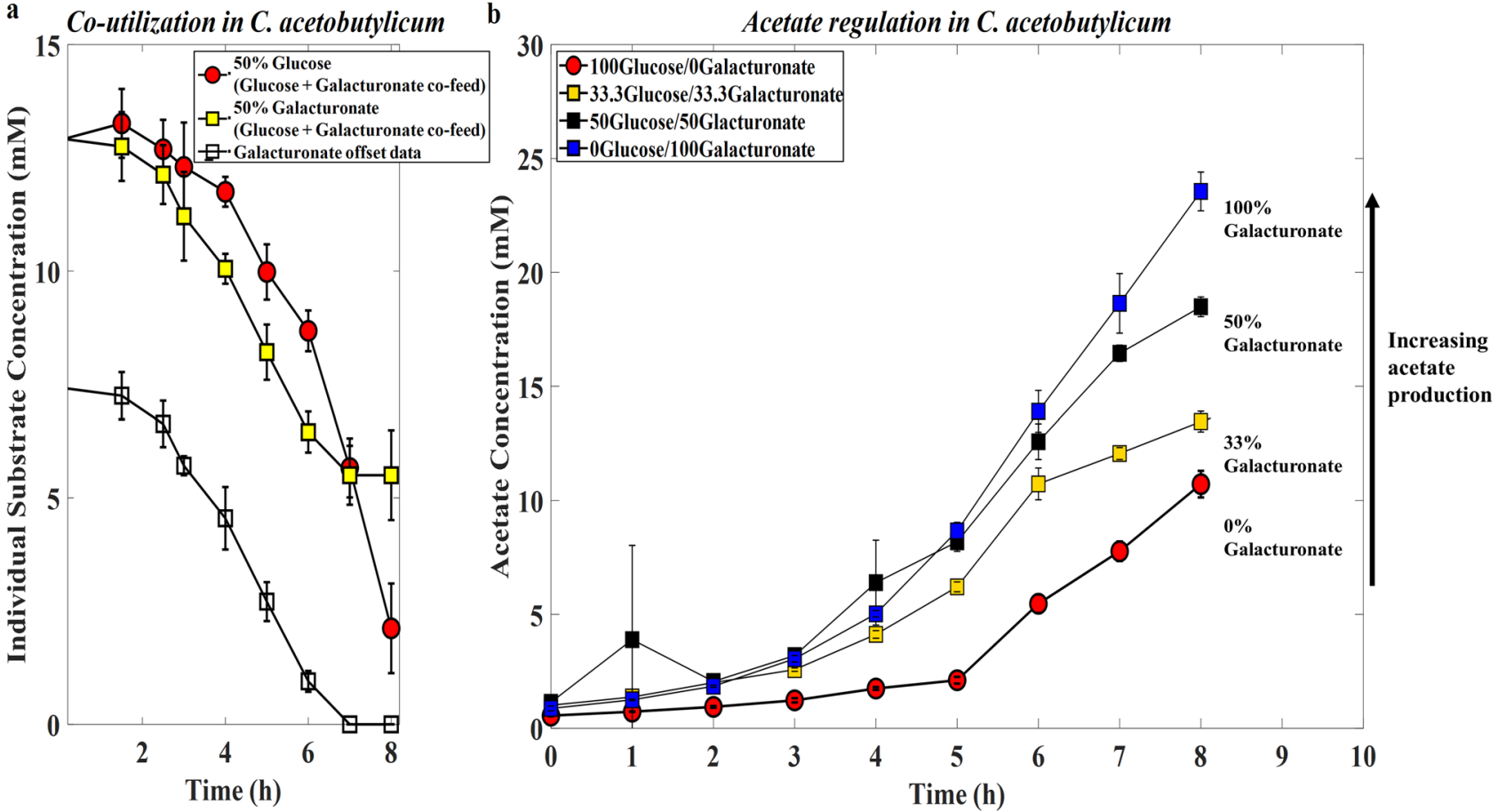

Figure 2. (a) Individual substrate consumption when C. acetobutylicum is co-fed a mixture of glucose and galacturonate. (b) Acetate regulation in C. acetobutylicum based on glucose-galacturonate co-feeding and co-utilization. Error bars are standard deviations of three biological repeat experiments.

When C. acetobutylicum was co-fed equal amounts of two substrates (Table 1-substrates \#3, \#7, and \#11), growth was substrate dependant (Supplementary Figure S1b, c). Metabolite data (Supplementary Figure S2d) suggest co-feeding equal portions of glucose with gluconate was the less preferred of the two-substrate mixtures, yielding approximately equal proportions of acetate and butyrate at $~ 7.5 \mathrm{mM}$. However, in co-feeds of equal amounts of glucose with galacturonate (Supplementary Figure S2), approximately twice the amount of acetate $(\sim 17 \mathrm{mM})$ was produced compared to butyrate $(\sim 7 \mathrm{mM})$. Co-feeding equal amounts of galacturonate with gluconate (Supplementary Figure S2.) produced $\sim 23 \mathrm{mM}$ acetate as the main product, comparative to yield from galacturonate only (Supplementary Figure S2c) even though a more considerable amount of the mixed substrate was consumed ( $\sim 25 \mathrm{mM}$ vs. $\sim 15 \mathrm{mM}$ from before). When equal proportions of all three substrates were co-fed to C. acetobutylicum (substrate \#13, Supplementary Figure S2g), suppressed growth was observed (data not included), and produced yields of $\sim 13 \mathrm{mM}$ acetate and $\sim 6 \mathrm{mM}$ butyrate.

Substrate co-utilization and product regulation in C. acetobutylicum. Careful examination of the results from the two-substrate growth curves (Supplementary Figure S1b, c), in comparison with individual substrate growth (Supplementary Figure S1a), suggested possible co-utilization of glucose and galacturonate. New experiments were conducted along-side repeat analyses to confirm this hypothesis. Samples were also collected for HPAEC-PAD analyses to determine the individual consumption profile of the different substrates in each mixture. The results presented in Fig. 2a below suggest that during the first $7 \mathrm{~h}$ of fermentation, C. acetobutylicum cells simultaneously co-utilized both glucose and galacturonate until the latter's exhaustion from the mixture. C. acetobutylicum used all of the galacturonate to completion first when mixed with a more preferred substrate, such as glucose. Combining this knowledge with the fact that galacturonate fermentations are nearly homoacetic, the metabolite data was re-analyzed for possible acetate regulation. Increasing the galacturonate to glucose percent $(\%)$ in the feed medium from 0,50 , and then 100 led to increased acetate production. For comparative reasons, co-utilization and acetate production in C. acetobutylicum are presented side-by-side in Fig. 2. As a positive control, on the same plot, we also plotted the acetate content in the three substrate mixture (\#13, 33.3\% galacturonate), where only a third of galacturonate was added. The results from Fig. $2 \mathrm{~b}$ confirmed an increase in the amount of acetate produced $(\sim 10 \mathrm{mM}, \sim 13 \mathrm{mM}, \sim 17 \mathrm{mM}$, and $\sim 23 \mathrm{mM})$ with increasing galacturonate content.

Fine-tuning metabolite output through substrate co-feeding. The experiments were repeated for two additional species of clostridia, C. saccharoperbutylacetonicum and C. beijerinckii, to determine whether the observed acetate regulation from glucose-galacturonate co-feeding was species-dependent. We also increased our testing ratios by addition of mid-points (25:75 and 75:25) between exclusive substrate additions ( 0 and 100), and equal mixtures (50:50) (Table 1-substrates \# 2, \#4, \#8, \#10 and \#12). Herein also, we extended the experiment time to capture phase transitions from acidogenesis into solventogenesis. $\mathrm{OD}_{600}$ measurements for the two species and conditions are presented in Supplementary Figure S1 alongside replicate growth curves for C. ace- 

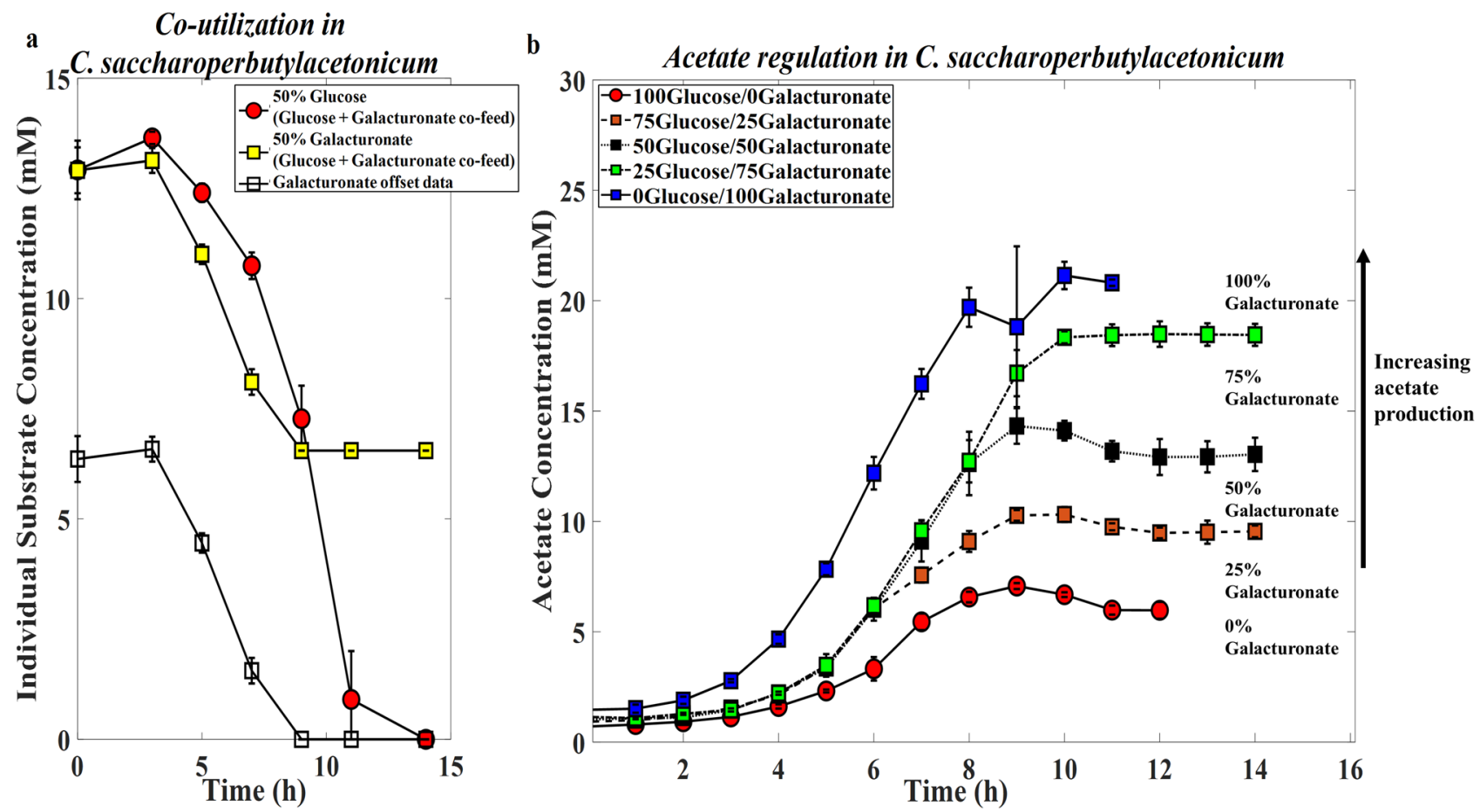

Figure 3. (a) Individual substrate consumption when C. saccharoperbutylacetonicum is co-fed a mixture of glucose and galacturonate. (b) Acetate regulation in C. saccharoperbutylacetonicum based on glucosegalacturonate co-feeding and co-utilization. Error bars are standard deviations of three biological repeat experiments.

tobutylicum in the same plot. For both strains, again, glucose had the highest consumption rate (Supplementary Figures S2,S3). In contrast, however, to C. acetobutylicum fermentation discussed above, galacturonate was consumed more readily than gluconate in exclusive feeds. In exclusive galacturonate experiments, again, fermentations were nearly homoacetic. Also, the two strains appeared intolerant of gluconate as a substrate, producing insignificant amounts of metabolic products.

The metabolite data from exclusive glucose fermentation ( $30 \mathrm{mM}$ consumed, Supplementary Figure S3a), show that $C$. saccharoperbutylacetonicum produced near equal proportions of acetate $(\sim 6 \mathrm{mM})$ and butyrate $(\sim 5 \mathrm{mM})$ during acidogenesis, switching to high butanol production $(\sim 17.5 \mathrm{mM})$ in the second phase of the fermentation (solventogenic). During exclusive gluconate fermentations ( $\sim 0.5 \mathrm{mM}$ consumed, Supplementary Figure S3b), C. saccharoperbutylacetonicum barely consumed the available substrate, producing minimal fermentation products $(\sim 3.5 \mathrm{mM}$ butyrate, and $\sim 2.5 \mathrm{mM}$ acetate). However, in exclusive galacturonate fermentations ( $17.5 \mathrm{mM}$ consumed, Supplementary Figure S3c), C. saccharoperbutylacetonicum fermentation was mostly homoacetic yielding $\sim 21 \mathrm{mM}$ acetate. When C. saccharoperbutylacetonicum was co-fed equal proportions of two substrate mixtures (all substrates minus \#1, \#5, and \#9), growth was again substrate dependant (Supplementary Figure S1e,f). In co-fed fermentations with glucose-gluconate (Supplementary Figure S1e), growth was similar to exclusively fed glucose fermentation (Supplementary Figure S1d). The comparative metabolite data for the two-substrate mixtures (Supplementary Figure S3) for C. saccharoperbutylacetonicum are as follows: in general, for substrate mixtures with glucose-gluconate (Supplementary Figure S3d-f) no co-utilization was observed (data not shown), and acetate regulation, if any, was at a minimum from $\sim 5 \mathrm{mM}$ to $\sim 7.5 \mathrm{mM}$ during the experiment as the gluconate percent content was increased from 25 to 50 , and then 75 . In co-feeds with glucose-galacturonate (Supplementary Figure S3g-i), co-utilization of glucose and galacturonate was observed within the first $9 \mathrm{~h}$ of fermentation (Fig. 3a). Acetate yields increased (Fig. 3b) from $\sim 7 \mathrm{mM}$ to $\sim 10 \mathrm{mM}, \sim 14 \mathrm{mM}, \sim 19 \mathrm{mM}$, through to $\sim 21 \mathrm{mM}$ as the galacturonate percent content in the feed was increased from 0 to $25,50,75$, and 100 respectively. In all co-fed two substrate mixtures (Supplementary Figure S3d-i), the more oxidized substrates likely failed to produce the conditions needed for solventogenesis due to the lower availability of NADH. Substrate combinations with overall lower oxidation numbers were more favorable for butanol production. For example, glucose-gluconate mixtures were more favorable compared to glucose-galacturonate mixtures.

In C. beijerinckii, the metabolite data for exclusive glucose fermentation $(\sim 20 \mathrm{mM}$ consumed, Supplementary Figure S4a), show that $C$. beijerinckii produced mostly butyrate $(\sim 17.5 \mathrm{mM})$ during the $14 \mathrm{~h}$ of fermentation. During exclusive gluconate fermentation ( $\sim 0.5 \mathrm{mM}$ consumed in the first $12 \mathrm{~h}$, Supplementary Figure S4b), $C$. beijerinckii consumed very minimal substrates and produced insignificant amounts of fermentation products (recorded below $\sim 3 \mathrm{mM}$ on average). Exclusive galacturonate fermentation ( $\sim 16 \mathrm{mM}$ consumed, Supplementary Figure S4c), for this same strain, was mostly homoacetic ( $\sim 29 \mathrm{mM}$ acetate produced). When C. beijerinckii was co-fed two substrate mixtures (all substrates minus \#1, \#5, and \#9), growth was generally comparable to growth during exclusive glucose fermentation (Supplementary Figure S1h, i). The comparative metabolite data for the 
a

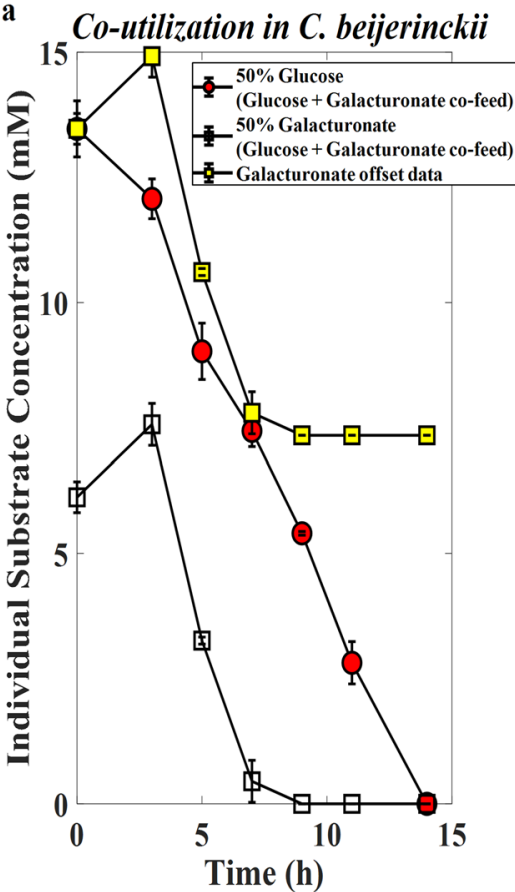

b

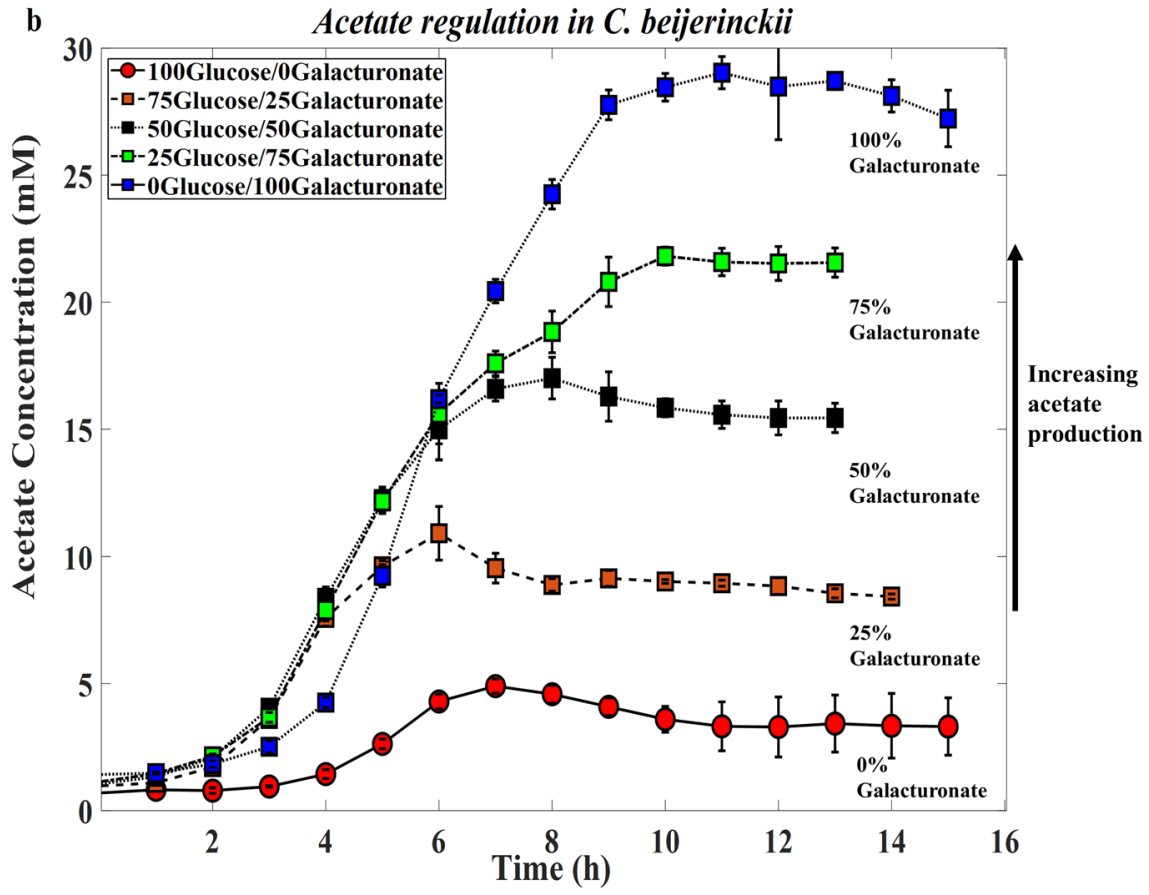

Figure 4. (a) Individual substrate consumption when C. beijerinckii is co-fed a mixture of glucose and galacturonate. (b) Acetate regulation in C. beijerinckii based on glucose-galacturonate co-feeding and co-utilization. Error bars are standard deviations of three biological repeat experiments.

two-substrate mixtures (Supplementary Figure S4) for C. beijerinckii are as follows: in general, substrate mixtures with glucose-gluconate (Supplementary Figure S4d-f), acetate regulation was not observed, and butyrate was the main product in all three instances. In substrate mixtures with glucose-galacturonate (Supplementary Figure S4g-i) co-utilization of glucose and galacturonate was observed within the first $7 \mathrm{~h}$ of fermentation (Fig. 4a) and acetate yields increased (Fig. $4 \mathrm{~b}$ ) from $\sim 4 \mathrm{mM}$ to $\sim 9 \mathrm{mM}, \sim 15 \mathrm{mM}, \sim 22 \mathrm{mM}$, and finally to $\sim 28 \mathrm{mM}$ as the galacturonate percent content in the fed was increased from 0 to $25,50,75$, and then 100 , respectively. No butanol production was observed for experiments with $C$. beijerinckii under all experimental conditions.

\section{Discussion}

This research builds on findings reported by our lab ${ }^{28}$ for agitated C. acetobutylicum fermentation on glucose, gluconate, and galacturonate. The agitation of fermentation cultures has been shown to reduce total dissolved gas levels in a system ${ }^{28}$, which is advantageous towards growth for glucose and gluconate runs but not for runs on galacturonate. In the work presented here, under agitated batch conditions, the three substrates were cofed in turns and together for three different Clostridium sp.-acetobutylicum, saccharoperbutylacetonicum, and beijerinckii.

For exclusive substrate feeds, glucose was the most readily used subsrate for all three Clostridium sp. However, fermentation on gluconate and galacturonate was species-dependent. Both C. saccharoperbutylacetonicum and C. beijerinckii consumed galacturonate but not gluconate, C. acetobutylicum consumed galacturonate and gluconate in these experiments and the earlier report for the same organism ${ }^{28}$. The species-dependent consumption of these substrates was further confirmed in the corresponding $\mathrm{OD}_{600}$ plots. The metabolite data for exclusively fed galacturonate fermentations supports the earlier results of homoacetic production for all three investigated strains ${ }^{28}$. Like before, for fermentations with glucose or gluconate, the production of butyrate (as the primary product) was species-dependent. Mainly, C. acetobutylicum produced butyrate under both substrate conditions. However, the other two clostridia strains only produced butyrate with glucose consumption and insignificant metabolite products for the less desirable substrate-gluconate-in this instance.

When galacturonate was co-fed with glucose its presence correlated to increased acetate production for all three species. The main finding in this work is the product regulation of acetate. In part, these results are supportive of research by Vasconcelos et al. $^{35}$, where metabolic output is affected by glucose-glycerol ratios in two-substrate co-feeds. In this very instance, glucose is the more oxidized substrate in comparison to glycerol.

Substrate co-utilization presents several advantages and opens up a vast region of exploration for control of chemical production in biological systems. Instead of access to discrete points shown in Fig. 1, substrate coutilization via manipulation of glucose and galacturonate in different proportions can enable advanced chemical production through a biological route. In addition to the three substrates (galacturonate, gluconate, and glucose) explored in this work, more substrates with different oxidation states (e.g., mannitol and glycerol) can be explored for possible co-utilization. The current findings show a proof of concept for continuous control of precusors for chemical production denoted by the plot in Fig. 5 above as opposed to discrete points which result from single substrates. For example, using C. acetobutylicum as a case study, co-feeding equal ratio of glucose 


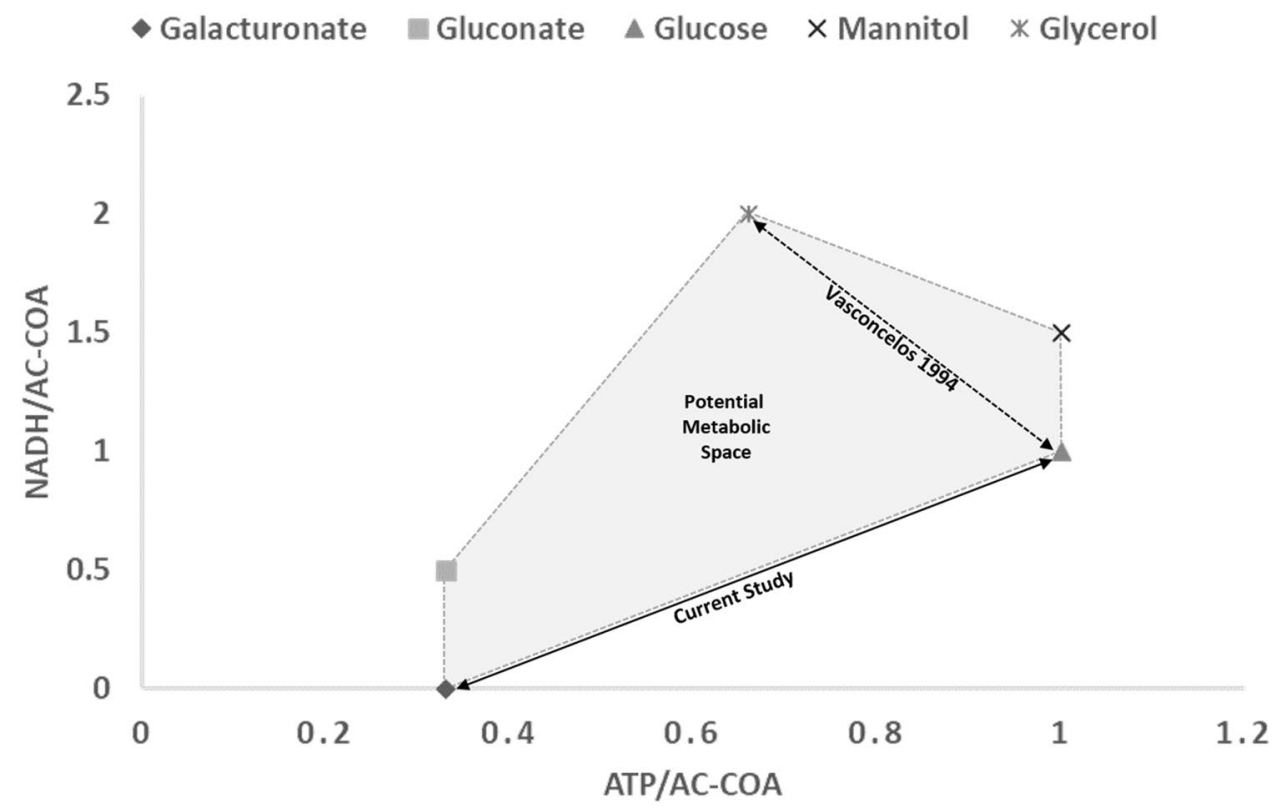

Figure 5. Proposed continuous exploration for chemical outputs from fermentations. Plot shows ratio of ATP/ $\mathrm{NADH}$ that is produced per each acetyl-CoA formed.

and galacturonate in the two-substrate mixtures had the same acetyl-CoA $(\sim 0.5)$ as exclusive feed $(\sim 0.5)$ on gluconate. However, it produced twice the amount of ATP $(\sim 0.7$ vs. $\sim 0.35)$. Since the redox recycling requirements remained unchanged, the acetate: butyrate ratios $(2: 1)$ are comparable in both instances (Supplementary Figure S2b,e). However, the carbon flux, and consequently, product yields differ under these two scenarios. The observed higher carbon flux results in more biomass due to ATP yields and subsequently lower products for the co-fed equal ratio of glucose and galacturonate. In exclusive gluconate feeds, however, higher product yields result in less biomass, likely because more subsrate is needed to make equivalent ATP. The directional carbon flux demonstrates another dimension of metabolic control beyond the redox changes explained via the acetate and butyrate ratio. By extending this analogy, a similar amount of ATP $(\sim 0.7)$ was produced for equal ratio co-feeds of glucose with either gluconate or galacturonate. However, NADH/acetyl-coA requirements were different $(\sim 0.5$ vs. $\sim 0.75)$. The change in redox recycling requirements is reflected in the acetate:butyrate ratios produced (1:1 vs. 2:1), as shown in Supplementary Figure S2,e. For tri-equal co-feed for galacturonate, gluconate, and glucose (33:33:33), NADH/acetyl-coA requirements $(\sim 0.5)$, and associated ATP production $(\sim 0.7)$ was similar to that for equal ratio co-feed of glucose and galacturonate. Again we see similar or un-changed acetate:butyrate ratio (2:1-Supplementary Figure S2g).

\section{Conclusion}

In order to bypass limitations on the degree of metabolic freedom, researchers should embrace the more complex bio-products synthesis employing multiple substrates in a fermentation. Traditionally, single substrates have been employed but have been shown to be metabolically wasteful and, in most instances, produce undesirable products, thus depriving the cell of precious resources that could be re-directed toward growth and maintenance. In this research, we show that desirable metabolic products can be regulated and optimized by co-feeding a mixture of substrates with different oxidation states to Clostridium sp., where substrates co-utilization is observed.

We present an exploratory region for five substrates with varying degrees of oxidations: galacturonate, gluconate, glucose, mannitol, and glycerol and show that acetate: butyrate ratios can be regulated through co-feeding (if co-utilization is present in the chosen organism). Likewise, we demonstrate another dimension of metabolic control beyond the redox, where carbon flux can be tuned toward higher product yields based on NADH/acetylCoA and ATP prevalence.

Future discoveries of co-utilization of other substrate mixes (e.g., for the five substrates listed above) can further open up this exploratory region. Additionally, different anaerobic fermentating bacteria can also be explored as candidates for this space. In silico modeling and simulations can be used together with a design of experiments to minimize the cost and time needed to narrow down potential candidates for subsequent testing and practical validation in the laboratory.

\section{Materials and methods}

Experimental design. Three (3) substrates: glucose, galacturonate, and gluconate were chosen as feedstock for fermentation experiments based on their oxidation states and reported metabolites. Clostridia cells were grown on the three individual substrates and several combinations of the substrates, as shown in Table 1 above. 


\begin{tabular}{|l|r|r|l|}
\hline Time (min) & \%A & \%B & \%water \\
\hline 00.00 & 80 & 0 & 20 \\
\hline 20.00 & 80 & 0 & 20 \\
\hline 50.00 & 0 & 100 & 0 \\
\hline 60.00 & 0 & 100 & 0 \\
\hline 61.00 & 80 & 0 & 20 \\
\hline 75.00 & 80 & 0 & 20 \\
\hline
\end{tabular}

Table 2. HPAEC-PAD separation parameters. A: $15 \mathrm{mM} \mathrm{NaOH}$; B: $150 \mathrm{mM} \mathrm{NaOAc}$ in $15 \mathrm{mM} \mathrm{NaOH}$. Flow rate: $1 \mathrm{~mL} / \mathrm{min}$. Column temperature: room temperature.

Bacteria strain maintenance and anaerobic culture conditions. Clostridium acetobutylicum ATCC 824, Clostridium saccharoperbutylacetonicum ATCC 27021 and Clostridium beijerinckii ATCC 25752 were purchased from the American Type Culture Collection (ATCC) and used in all experiments. Strains were maintained as spore stocks in a suspension of potato glucose medium containing per liter DI water: potato (grated), $150.0 \mathrm{~g}$; Glucose, $10.0 \mathrm{~g} ;\left(\mathrm{NH}_{4}\right)_{2} \mathrm{SO}_{4}, 0.5 \mathrm{~g}$; and $\mathrm{CaCO}_{3}, 3.0 \mathrm{~g}$ combined, then boiled $1 \mathrm{~h}$ mixing every $10 \mathrm{~min}$, filtered through cheesecloth, and autoclaved $120^{\circ} \mathrm{C} 10 \mathrm{~min}$ exposure ${ }^{11}$. Spore stocks were stored at room temperature in an anaerobic chamber (Coy Lab Products, MI) containing an atmosphere of approximately $90 \% \mathrm{~N}_{2}$, $5 \% \mathrm{CO}_{2}$, and $5 \% \mathrm{H}_{2}$.

Clostridial Growth Medium (CGM) was composed of the following components per liter of DI water: $\mathrm{KH}_{2} \mathrm{PO}_{4}, 0.75 \mathrm{~g} ; \mathrm{K}_{2} \mathrm{HPO}_{4}, 0.75 \mathrm{~g} ; \mathrm{MgSO}_{4} \cdot \mathrm{H}_{2} \mathrm{O}, 0.4 \mathrm{~g} ; \mathrm{MnSO}_{4}, 0.01 \mathrm{~g} ; \mathrm{FeSO}_{4} \cdot 7 \mathrm{H}_{2} \mathrm{O}, 0.01 \mathrm{~g} ; \mathrm{NaCl}, 1.0 \mathrm{~g} ; \mathrm{L}$-asparagine, 2.0 g; yeast extract, $5.0 \mathrm{~g}$; $\left(\mathrm{NH}_{4}\right)_{2} \mathrm{SO}_{4}, 2.0 \mathrm{~g}$; substrate (glucose, gluconate, or galacturonate), $5.0 \mathrm{~g}^{11}$. Chemicals used in medium preparation were purchased from Sigma Aldrich (St. Louis, MO, USA) and Becton Dickinson (Franklin Lakes, NJ, USA) and of HPLC grade (99\%), or extract suitable for use in culture media preparations.

Clostridium sp. was initially propagated on CGM under anaerobic conditions described previously ${ }^{28,40} .500$ $\mu \mathrm{L}$ of potato-glucose suspension (PGM) spore stock was heat-shocked at $80^{\circ} \mathrm{C}$ for $10 \mathrm{~min}$, then re-suspended in $150 \mathrm{~mL}$ CGM enriched with $0.5 \%$ of the respective carbohydrate (i.e., glucose, galacturonate, gluconate, or mixtures two or three of these substrates).

HPLC sample acquisition and analysis-individual sugars and metabolites. Cell growth experiments were conducted in triplicate for each of the three species investigated. $1 \mathrm{~mL}$ samples were extracted from experimental cultures hourly for $\mathrm{OD}_{600}$ measurements (Ultrospec 10/Amersham Biosciences). An additional $1 \mathrm{~mL}$ sample extracted, sterile filtered through a $0.2 \mu \mathrm{m}$ syringe filter (Corning), then stored at $+4^{\circ} \mathrm{C}$ for metabolite analysis via HPLC. HPLC analysis for the metabolic output was completed using an Agilent 1200 equipped with a refractive index detector and an Aminex HPX-87H cation exchange column $(300 \times 7.8 \mathrm{~mm}$ i.d. $\times 9 \mu \mathrm{m}$; Bio-Rad $)^{41} .100 \mu \mathrm{L}$ of each sample was injected into the system for isocratic elution, with a mobile phase of $3.25 \mathrm{mM} \mathrm{H}_{2} \mathrm{SO}_{4}$ at $0.6 \mathrm{~mL} / \mathrm{min}$ and $35^{\circ} \mathrm{C}$.

HPLC sample acquisition and analysis-sugar mixtures. In order to confirm the co-utilization of glucose with galacturonate, new separation methods for detection were developed and used. The sugar mixtures were separated by high-performance anion-exchange chromatography with pulsed amperometric detection (HPAEC-PAD). The chromatography was carried out on a Dionex DX-600 system consisting of an AS50 autosampler, LC30 column compartment, GP50 gradient pump, and ED50 electrochemical detector (Dionex/ Thermo Fisher Scientific). The column is CarboPac PA1 $(4 \mathrm{~mm} \times 250 \mathrm{~mm})$. The injection volume is $10 \mu \mathrm{L}$. The gradient is shown below in Table 2 .

Received: 15 June 2020; Accepted: 19 October 2020

Published online: 08 January 2021

\section{References}

1. Staniek, A. et al. Natural products-learning chemistry from plants. Biotechnol. J. 9(3), 326-336. https://doi.org/10.1002/biot.20130 0059 (2014).

2. Zhang, Y.-H.P. Simpler is better: High-yield and potential low-cost biofuels production through cell-free synthetic pathway biotransformation (sypab). Acs Catal. 1(9), 998-1009. https://doi.org/10.1021/cs200218f (2011).

3. Basen, M. et al. Single gene insertion drives bioalcohol production by a thermophilic archaeon. Proc. Natl. Acad. Sci. 111(49), 17618-17623. https://doi.org/10.1073/pnas.1413789111 (2014).

4. Peralta-Yahya, P. P., Zhang, F., Del Cardayre, S. B. \& Keasling, J. D. Microbial engineering for the production of advanced biofuels. Nature 488(7411), 320-328. https://doi.org/10.1038/nature11478 (2012).

5. Xu, L., Weathers, P. J., Xiong, X. R. \& Liu, C. Z. Microalgal bioreactors: Challenges and opportunities. Eng. Life Sci. 9(3), 178-189. https://doi.org/10.1002/elsc.200800111 (2009).

6. Liu, Y., Cao, Y., Du, G. \& Liu, L. Systems and synthetic metabolic engineering: Challenges and prospects. In Systems and Synthetic Metabolic Engineering 237-264 (Academic Press, New York, 2020). https://doi.org/10.1016/B978-0-12-821753-5.00010-1. 
7. Cao, M., Tran, V. G. \& Zhao, H. Unlocking nature’s biosynthetic potential by directed genome evolution. Curr. Opin. Biotechnol. 66, 95-104. https://doi.org/10.1016/j.copbio.2020.06.012 (2020).

8. Smanski, M. J. et al. Synthetic biology to access and expand nature's chemical diversity. Nat. Rev. Microbiol. 14(3), 135. https://doi. org/10.1038/nrmicro.2015.24 (2016).

9. Santos-Merino, M., Singh, A. K. \& Ducat, D. C. New applications of synthetic biology tools for cyanobacterial metabolic engineering. Front. Bioeng. Biotechnol. 7, 33 (2019).

10. Wu, G. et al. Metabolic burden: Cornerstones in synthetic biology and metabolic engineering applications. Trends Biotechnol. 34(8), 652-664. https://doi.org/10.1016/j.tibtech.2016.02.010 (2016).

11. Adler, H. I. \& Crow, W. A technique for predicting the solvent-producing ability of Clostridiumacetobutylicum. Appl. Environ. Microbiol. 53(10), 2496-2499 (1987).

12. Navarrete, C., Jacobsen, I. H., Martínez, J. L. \& Procentese, A. Cell factories for industrial production processes: Current issues and emerging solutions. Processes 8(7), 768 (2020).

13. Du, J., Shao, Z. \& Zhao, H. Engineering microbial factories for synthesis of value-added products. J. Ind. Microbiol. Biotechnol. 38(8), 873-890. https://doi.org/10.1007/s10295-011-0970-3 (2011).

14. Zhang, X. et al. Modular pathway engineering of key carbon-precursor supply-pathways for improved N-acetylneuraminic acid production in Bacillussubtilis. Biotechnol. Bioeng. 115(9), 2217-2231 (2018).

15. Babel, W. The auxiliary substrate concept: From simple considerations to heuristically valuable knowledge. Eng. Life Sci. 9(4), 285-290 (2009).

16. Fernie, A. R. \& Bauwe, H. Wasteful, essential, evolutionary stepping stone? The multiple personalities of the photorespiratory pathway. Plant J. 102, 4 (2020).

17. Francois, J. M., Alkim, C. \& Morin, N. Engineering microbial pathways for production of bio-based chemicals from lignocellulosic sugars: Current status and perspectives. Biotechnol. Biofuels 13(1), 1-23 (2020).

18. Varma, A. \& Palsson, B. O. Metabolic capabilities of Escherichiacoli: I: Synthesis of biosynthetic precursors and co-factors. J. Theor. Biol. 165(4), 477-502 (1993).

19. Agu, C.V., Ujor, V., \& Ezeji, T. C. Metabolic engineering of clostridium beijerinckii to improve glycerol metabolism and furfural tolerance. $9 / 11$

20. Xin, X., Cheng, C., Du, G., Chen, L. \& Xue, C. Metabolic engineering of histidine kinases in Clostridium beijerinckii for enhanced butanol production. Front. Bioeng. Biotechnol. 8, 214. https://doi.org/10.3389/fbioe.2020.00214 (2020).

21. Procentese, A. et al. Continuous xylose fermentation by Clostridiumacetobutylicum-assessment of solventogenic kinetics. Bioresour. Technol. 192, 142-148. https://doi.org/10.1016/j.biortech.2015.05.041 (2015).

22. Ma, Y. \& Liu, Y. Turning food waste to energy and resources towards a great environmental and economic sustainability: An innovative integrated biological approach. Biotechnol. Adv. 37, 7 (2019).

23. Dominguez, T. Characterizing emissions from open burning of military food waste and packaging from forward operating bases. (2016).

24. Rock, K., et al. An Analysis of Military Field-Feeding Waste (No. NATICK/TR-00/021). Army Soldier and Biological Chemical Command Natick Ma Soldier Systems Center (2000).

25. Knowlton, L., Pickard, D., Diebold, J., Lasnik, K., Lilley, A., \& Browne, K. On-Site Field-Feeding Waste to Energy Converter. Army Natick Soldier Research Development and Engineering Center Ma (2008).

26. Maitan-Alfenas, G. P., Visser, E. M. \& Guimarães, V. M. Enzymatic hydrolysis of lignocellulosic biomass: Converting food waste in valuable products. Curr. Opin. Food Sci. 1, 44-49. https://doi.org/10.1016/j.cofs.2014.10.001 (2015).

27. Huisjes, E. H. et al. Toward pectin fermentation by Saccharomycescerevisiae: Expression of the first two steps of a bacterial pathway for d-galacturonate metabolism. J. Biotechnol. 162(2-3), 303-310 (2012).

28. Servinsky, M. D. et al. Fermentation of oxidized hexose derivatives by Clostridiumacetobutylicum. Microb. Cell Factor. $13(1), 139$. https://doi.org/10.1186/s12934-014-0139-7 (2014).

29. Lütke-Eversloh, T. \& Bahl, H. Metabolic engineering of Clostridiumacetobutylicum: Recent advances to improve butanol production. Curr. Opin. Biotechnol. 22(5), 634-647. https://doi.org/10.1016/j.copbio.2011.01.011 (2011).

30. Lütke-Eversloh, T. Application of new metabolic engineering tools for Clostridiumacetobutylicum. Appl. Microbiol. Biotechnol. 98(13), 5823-5837. https://doi.org/10.1007/s00253-014-5785-5 (2014).

31. Stephanopoulos, G. Bioprocess and microbe engineering for total carbon utilization in biofuel production. Mass. Inst. Technol. 2011. U.S. Pat. Appl. 13/007,325.

32. Wen, Z., Ledesma-Amaro, R., Lu, M., Jin, M. \& Yang, S. Metabolic engineering of Clostridiumcellulovorans to improve butanol production by consolidated bioprocessing. ACS Synth. Biol. 9(2), 304-315 (2020).

33. Li, A. et al. Developing Clostridiumdiolis as a biorefinery chassis by genetic manipulation. Bioresour. Technol. 305, 123066 (2020).

34. Desai, R. P., Harris, L. M., Welker, N. E. \& Papoutsakis, E. T. Metabolic flux analysis elucidates the importance of the acid-formation pathways in regulating solvent production by Clostridiumacetobutylicum. Metab. Eng. 1(3), 206-213. https://doi.org/10.1006/mben. 1999.0118 (1999).

35. Vasconcelos, I. S. A. B. E. L., Girbal, L. \& Soucaille, P. Regulation of carbon and electron flow in Clostridiumacetobutylicum grown in chemostat culture at neutral $\mathrm{pH}$ on mixtures of glucose and glycerol. J. Bacteriol. 176(5), 1443-1450. https://doi.org/10.1128/ jb.176.5.1443-1450.1994 (1994).

36. Wubben, J. P., ten Have, A., van Kan, J. A. \& Visser, J. Regulation of endopolygalacturonase gene expression in Botrytiscinerea by galacturonic acid, ambient $\mathrm{pH}$ and carbon catabolite repression. Curr. Genet. 37(2), 152-157. https://doi.org/10.1007/s002940050 $022(2000)$

37. Collmer, A. \& Bateman, D. Impaired induction and self-catabolite repression of extracellular pectate lyase in Erwiniachrysanthemi mutants deficient in oligogalacturonide lyase. Proc. Natl. Acad. Sci. 78(6), 3920-3924. https://doi.org/10.1073/pnas.78.6.3920 (1981).

38. Aguilar, G. \& Huitron, C. Stimulation of the production of extracellular pectinolytic activities of Aspergillus sp. by galacturonic acid and glucose addition. Enzym. Microb. Technol. 9(11), 690-696. https://doi.org/10.1016/0141-0229(87)90129-3 (1987).

39. Behrens, S., Bahl, H. \& Mitchell, W. J. Molecular analysis of the mannitol operon of Clostridium acetobutylicum encoding a phosphotransferase system and a putative PTS-modulated regulator. Microbiology 147(1), 75-86 (2001).

40. Zu, T. N. et al. Real-time metabolite monitoring of glucose-fed Clostridiumacetobutylicum fermentations using Raman assisted metabolomics. J. Raman Spectrosc. 48(12), 1852-1862. https://doi.org/10.1002/jrs.5264 (2017).

41. $\mathrm{Zu}, \mathrm{T}$. N. et al. Predictive modeling in Clostridiumacetobutylicum fermentations employing Raman spectroscopy and multivariate data analysis for real-time culture monitoring. Int. Soc. Opt. Photon. 9863, 98630I. https://doi.org/10.1117/12.2228545 (2016).

\section{Acknowledgements}

The authors acknowledge support for W. Mojadedi from the U.S. Army Research Laboratory Journeyman Fellowship Program administered by the Oak Ridge Associated Universities. 


\section{Author contributions}

T.N.K.Z. and C.J.S. conceived the study. E.S.G. facilitated all bioreactor set-up and operation. T.N.K.Z. and E.S.G. monitored, and collected all requisite samples for analysis. S.L. and W.M. implemented HPLC metabolite analysis. T.N.K.Z., S.L., and C.J.S. analyzed data. T.N.K.Z. led writing of manuscript. S.L. and C.J.S. contributed to writing the manuscript. All authors have read and provided edits to the final document.

\section{Competing interests}

The authors declare no competing interests.

\section{Additional information}

Supplementary information is available for this paper at https://doi.org/10.1038/s41598-020-76761-4.

Correspondence and requests for materials should be addressed to T.N.K.Z.

Reprints and permissions information is available at www.nature.com/reprints.

Publisher's note Springer Nature remains neutral with regard to jurisdictional claims in published maps and institutional affiliations.

(c) Open Access This article is licensed under a Creative Commons Attribution 4.0 International License, which permits use, sharing, adaptation, distribution and reproduction in any medium or format, as long as you give appropriate credit to the original author(s) and the source, provide a link to the Creative Commons licence, and indicate if changes were made. The images or other third party material in this article are included in the article's Creative Commons licence, unless indicated otherwise in a credit line to the material. If material is not included in the article's Creative Commons licence and your intended use is not permitted by statutory regulation or exceeds the permitted use, you will need to obtain permission directly from the copyright holder. To view a copy of this licence, visit http://creativecommons.org/licenses/by/4.0/.

This is a U.S. Government work and not under copyright protection in the US; foreign copyright protection may apply 2022 